\title{
Development of a Continuous Photochemical Benzyne-Forming Process
}

\author{
Cormac Bracken ${ }^{a}$ (D) \\ Andrei S. Batsanov (D) \\ Marcus Baumann*a (iD) \\ a School of Chemistry, University College Dublin, \\ Science Centre South, D04 N2E2, Dublin, Ireland \\ marcus.baumann@ucd.ie \\ b Department of Chemistry, University of Durham, \\ South Road, DH1 3LE, Durham, UK
}

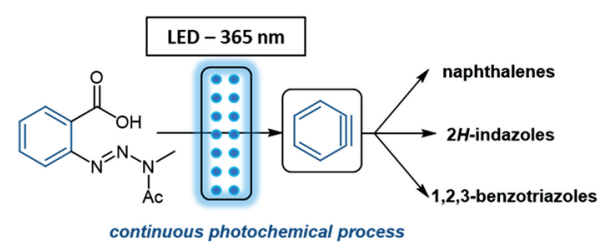

light LEDs as well as traditional UV lamps to create a multitude of important chemical entities. ${ }^{4}$ In view of the industrial applications, photochemical transformations are gaining momentum as photons are seen as traceless and tunable reagents, and recent reports on the scalability of continuous photochemical processes ${ }^{3 c, 5}$ including those towards drugs like artemisinin ${ }^{6}$ have highlighted the potential for their exploitation.

Given the salient features of flow chemistry, a class of important transformations that is noticeably underutilized, concerns the generation and use of benzynes. ${ }^{7}$ While Kobayashi's mild method starting from ortho-TMS aryl triflates $^{8}$ is one of the most exploited strategies for accessing benzynes, alternatives based on diazotization and subsequent fragmentation of anthranilic acid derivatives or by ortho-lithiation of halobenzenes, have been used. ${ }^{9}$ Although the last decades have witnessed impressive applications of especially Kobayashi's method, which include various studies towards heteroarynes, ${ }^{10}$ the generation and use of benzynes is not a commonly exploited strategy. This may point to the necessity for generating bespoke substrates (e.g., TMS-aryl triflates) that require fluoride as an activation reagent to trigger benzyne formation, as well as concerns regarding scale-up of the exothermic benzyne forming process.

We were thus intrigued by the opportunity to develop a continuous light-driven benzyne forming reaction that would overcome some of these limitations. Crucially, such a continuous approach would effectively exploit the benefits of flow chemistry, thereby rendering a robust method to enable and stimulate further developments in this important area of research.

Upon surveying the literature, we identified two suitable precursors that would render the desired benzyne species 1 upon irradiation with UV light (Scheme 1). 


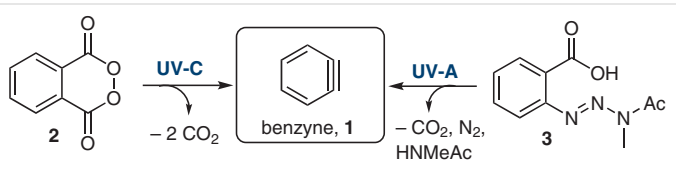

Scheme 1 Comparison of precursors for photochemical benzyne formation

Phthaloyl peroxide $\mathbf{2}$ is known to be a suitable benzyne precursor with recent exploitations demonstrating its use towards the creation of benzotriazoles. ${ }^{11}$ Benzoic acid derived triazine $\mathbf{3}$ was initially reported as a photolysis product from nitro benzaldehyde $\mathrm{N}$-acylhydrazones that would subsequently generate benzyne. ${ }^{12}$ Whilst both species would generate benzyne accompanied by liberation of gaseous by-products $\left(\mathrm{CO}_{2}\right.$ and $\left.\mathrm{N}_{2}\right)$, we decided to exploit the latter option as a recent report by Schnarr ${ }^{13}$ outlines the use of milder UV-A light in combination with short reaction times, which appeared preferable in view of broader substrate scope and fast reactions.

Following the reported synthesis ${ }^{13}$ of triazine species 3 that exploits diazotization of 2-aminobenzylalcohol (4), sequential trapping of the diazonium intermediate with methyl amine and acetyl chloride and final oxidation to render the carboxylic acid, we were able to access gram quantities of this building block (Scheme 2). Pleasingly, we were able to increase the overall yield of 3 by ca. $20 \%$ by modifying the isolation and purification process slightly (see below).

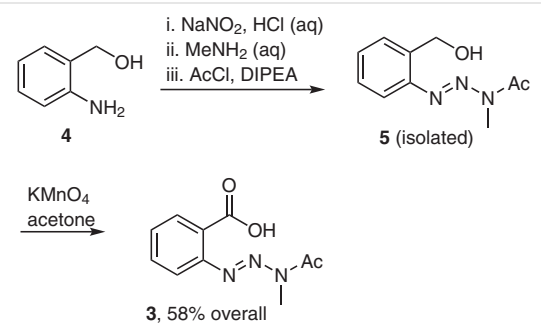

Scheme 2 Synthesis of benzyne precursor 3

Despite prior studies on this structure towards formation of benzyne, we were surprised by the lack of detailed characterization that would determine its suitability towards photochemical benzyne formation. To this end, we first recorded the UV/Vis spectrum of $\mathbf{3}$, which showed a maximum absorbance at $288 \mathrm{~nm}$ extending as anticipated as far as $370 \mathrm{~nm}$ (UV-A; Figure 1). This provided valuable information regarding the choice of light source in subsequent studies.

In addition, single crystals of $\mathbf{3}$ were obtained by slow evaporation of a sample solution (in DCM) allowing us to analyze its molecular structure by X-ray diffraction (Figure 2 ). This structure ${ }^{14}$ reveals a hydrogen bond in the solid state between the carboxyl group and the triazene moiety,

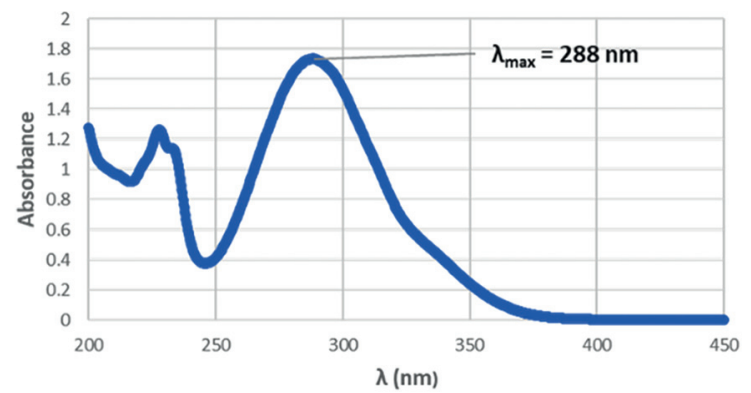

Figure 1 UV/Vis spectrum of benzyne precursor 3 (MeCN)

locking the conformation of this entity. Furthermore, this results in planarity for the entire scaffold, enabling efficient light absorption due to extended $\pi$-conjugation, which mirrors the information observed in the UV/Vis spectrum of $\mathbf{3}$.

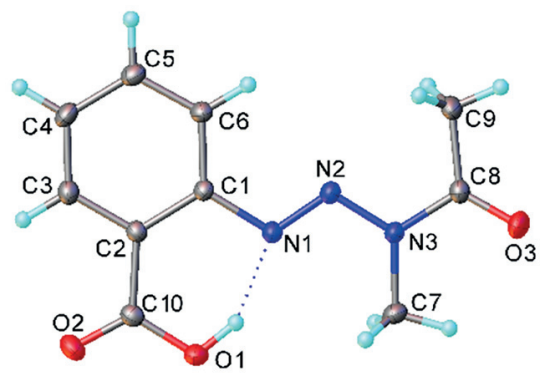

Figure 2 Molecular structure of 3

We next turned our attention to the development of a continuous-flow process that would exploit a photochemical reactor to generate the benzyne intermediate in the presence of various trapping agents. Specifically, we felt that flow processing would provide a superior approach, as the gaseous by-products are released safely and steadily (facilitated by using a backpressure regulator, BPR), whilst the uniform irradiation in combination with excellent temperature control and reaction automation are key features for future scale-up to create gram quantities of products. Based on our group's prior studies exploiting continuous photochemical approaches, we opted to utilize a Vapourtec E-series flow platform in combination with a tunable high-power LED (2 panels, $25-50 \mathrm{~W}$ each) emitting light at $365 \mathrm{~nm} .{ }^{15}$ The LED is thereby placed at the center of a flow coil reactor $(10 \mathrm{~mL}, \mathrm{FEP})$ and the two LED panels can be operated either separately or in combination, depending on the specific application (see the Supporting Information for photographic details). Temperature control is achieved by directing a stream of chilled gas ( $\mathrm{N}_{2}$ or compressed air) into the encased reactor system. Upon exiting this setup, the reaction mixture passes an adjustable BPR ( $0-10$ bar) prior to collection (Scheme 3). 


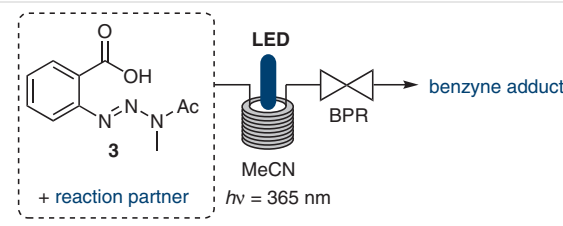

Scheme 3 Flow reactor setup for continuous benzyne formation

As depicted in Scheme 3, the benzyne precursor 3 and the desired reaction partner would be premixed as no reaction takes place in the absence of UV-light. To replicate the synthesis of previously reported benzotriazoles, ${ }^{13}$ which are versatile scaffolds of biological relevance, ${ }^{16}$ we prepared a solution of azide substrate ( 2 equiv) and benzyne precursor 3 ( 1 equiv, $\mathrm{MeCN}, 0.05 \mathrm{M}$ ) and pumped this into the high-power LED reactor to optimize power setting, temperature, and residence time. Pleasingly, it was quickly found that both benzyl azide and phenacetyl azide were competent reaction partners that generated the desired benzotriazole targets $\mathbf{6 a}$ and $\mathbf{6 b}$ within a short residence times of 3 minutes (Scheme 4). To minimize thermal degradation and potential over-irradiation, the reactor temperature was maintained at ca. $28{ }^{\circ} \mathrm{C}$ whilst limiting the LED power to $37.5 \mathrm{~W}$ using only one panel of LEDs. Due to the formation of gaseous by-products $\left(\mathrm{CO}_{2}\right.$ and $\left.\mathrm{N}_{2}\right)$ during the benzyne forming step, a slug-flow pattern was observed in the tubing. Separation of these gases was achieved upon collection of the crude product after passing a BPR (ca. 2 bar).

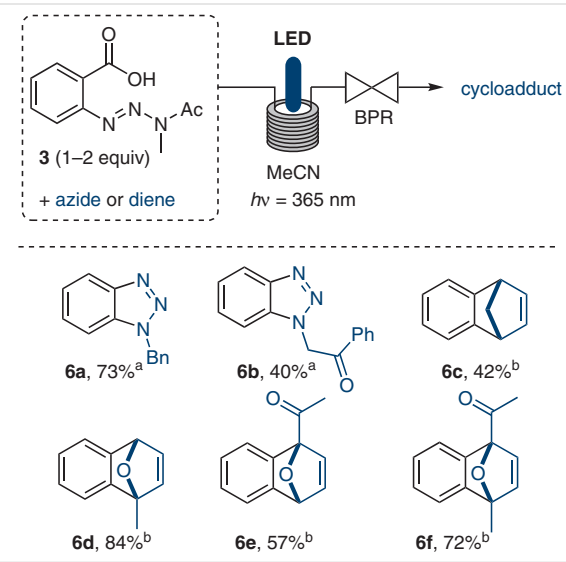

Scheme 4 Flow synthesis of benzotriazoles and other cycloadducts; 1.0 equiv of 3 ; ${ }^{b} 2.0$ equiv of 3 .

To further exploit this continuous photochemical benzyne forming process, we wished to also study a set of cyclic dienes as reaction partners. We thus selected cyclopentadiene and various furans and subjected their solutions with 3 (MeCN, $50 \mathrm{mM}$ ) to our flow process. As indicated through initial test reactions, higher yields of the corresponding cycloadducts $\mathbf{6 c - f}$ were achieved when using an excess of 3 (2 equiv). As before, the use of the LED emitting at $365 \mathrm{~nm}$ was key to the successful synthesis of the desired cycloadducts and enabled fast reactions through a short residence time of 3 minutes.

Having demonstrated proof of concept, we next wished to evaluate our method for the synthesis of $2 \mathrm{H}$-indazoles that are frequently exploited heterocycles in medicinal chemistry programs. ${ }^{17}$ As their conventional synthesis relies on multistep routes, we felt that a flow process could streamline this effort. Specifically, we wished to utilize sydnones ${ }^{18}$ as readily accessible mesoionic reaction partners that would initially undergo a formal [3+2]-cycloaddition with benzyne, followed by [4+2]-cycloreversion. The release of gaseous $\mathrm{CO}_{2}$ would thereby be easily accommodated within our flow process.

The synthesis of the sydnones was accomplished by following literature precedent, ${ }^{18}$ converting $\mathrm{N}$-phenyl glycine derivatives and proline methyl ester via $N$-nitrosated intermediates (8) into the corresponding building blocks (Scheme 5, see the Supporting Information for details).

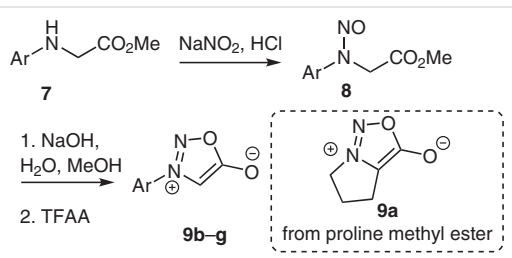

Scheme 5 Synthesis of sydnones $\mathbf{9 a}-\mathbf{g}$

With a small selection of sydnones at hand, we next embarked on their transformation into $2 H$-indazoles. As before, a stock solution of benzyne precursor $(3,2$ equiv, $0.1 \mathrm{M}$ $\mathrm{MeCN}$ ) and sydnone (1 equiv) was prepared and pumped through the photoreactor $(365 \mathrm{~nm}, 37.5 \mathrm{~W})$ with a residence time of 3 minutes. Pleasingly, the formation of several $2 \mathrm{H}$-indazoles was achieved in isolated yields up to $82 \%$ (Scheme 6 ). Whilst the proline derived sydnone (9a) and various electron-rich aryl appendages on the sydnone precursor enabled high yields (68-82\%), we noticed that electron-withdrawing substituents (e.g., 3,4-dichlorophenyl) reduced the yield significantly. ${ }^{19}$ Furthermore, using 2,6-dimethylphenyl-substituted sydnone, no $2 \mathrm{H}$-indazole product (10g) was observed. Whilst unexpected at first, we surmise that this substitution pattern leads to twisting of both rings, as indicated by a significant drop in the UV-absorbance (see the Supporting Information for details). The loss of conjugation arising from this conformational change thus appears to cause the observed reaction outcome.

To test the robustness and scalability of this flow process towards the creation of $2 \mathrm{H}$-indazole species, we selected proline derived sydnone 9a as the substrate and processed $760 \mathrm{mg}$ through our LED flow reactor. Pleasingly, this resulted in the generation of $648 \mathrm{mg}$ of the desired target 


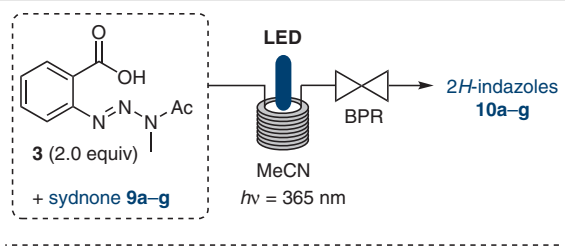<smiles></smiles>
$10 \mathrm{a}, 68 \%$<smiles>CCc1ccc2cn(-c3ccc(C)cc3)nc2c1</smiles>
$10 b, \mathrm{R}=\mathrm{H}, 77 \%$ $10 \mathrm{c}, \mathrm{R}=\mathrm{Me}, 82 \%$ Od, $\mathrm{R}=\mathrm{OMe}, 79 \%$<smiles>Clc1ccc(-n2cc3ccccc3n2)cc1Cl</smiles>
$10 \mathrm{e}, \mathrm{R}=\mathrm{i} \mathrm{Pr}, 70 \%$

$10 \mathrm{f}, 18 \%$

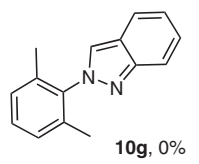

Scheme 6 Flow synthesis of $2 \mathrm{H}$-indazoles $10 \mathbf{a}-\mathbf{g}$ via benzyne cycloaddition cascade

( $68 \%$ yield) with a throughput of $4.4 \mathrm{mmol} / \mathrm{h}$. Furthermore, we secured single crystals of product 10a allowing us to verify its anticipated molecular structure by means of X-ray diffraction (Figure 3 ). ${ }^{20}$

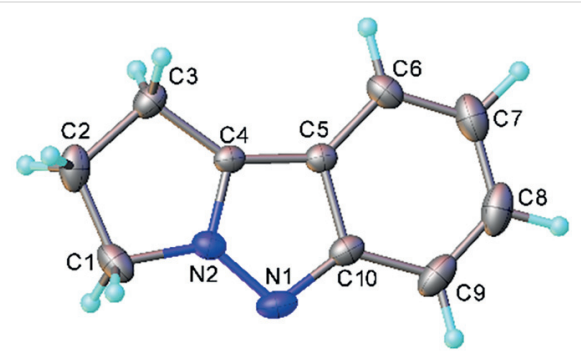

Figure 3 Molecular structure of 10a

In conclusion, we have developed a versatile flow protocol for the photochemical generation and concurrent trapping of benzyne from a stable and readily accessible triazine precursor. Crucially, the tunable high-power LED light source $^{21}$ provided for uniform irradiation at $365 \mathrm{~nm}$ and rendered a variety of important drug-like heterocycles in short residence times and in high yields. Lastly, the scalability of this flow process has been demonstrated, enabling and facilitating future exploitations of continuous benzynebased transformations.

Solvents were purchased from Sigma-Aldrich and used without further purification. Substrates and reagents were purchased from Alfa Aesar, Fischer, Fluorochem or Sigma-Aldrich and used as received. ${ }^{1} \mathrm{H}$ NMR spectra were recorded with 400 and $600 \mathrm{MHz}$ instruments and are reported relative to residual solvent: $\mathrm{CHCl}_{3}(\delta=7.26 \mathrm{ppm}) \cdot{ }^{13} \mathrm{C}$ NMR spectra were recorded with the same instruments (100 and 151 $\mathrm{MHz}$ ) and again are reported relative to $\mathrm{CHCl}_{3}(\delta=77.16 \mathrm{ppm})$. Data reported for ${ }^{1} \mathrm{H}$ NMR are as follows: chemical shift $(\delta / \mathrm{ppm})$ (multi- plicity, coupling constant (Hz), integration). Multiplicities are reported as follows: $\mathrm{s}=$ singlet, $\mathrm{d}=$ doublet, $\mathrm{t}=$ triplet, $\mathrm{q}=$ quartet, $\mathrm{p}=$ pentet, $\mathrm{h}=$ heptet, $\mathrm{m}=$ multiplet. Data for ${ }^{13} \mathrm{C}\left\{{ }^{1} \mathrm{H}\right\}$ NMR are reported in terms of chemical shift ( $\delta / \mathrm{ppm})$ and multiplicity $\left(\mathrm{C}, \mathrm{CH}, \mathrm{CH}_{2}\right.$, or $\left.\mathrm{CH}_{3}\right)$. COSY, HSQC and HMBC, experiments were used in the structural assignment. IR spectra were recorded with a Bruker Platinum spectrophotometer (neat, ATR sampling) with the intensities of the characteristic signals being reported as weak ( $w,<20 \%$ of the tallest signal), medium ( $\mathrm{m}, 21-70 \%$ of the tallest signal), or strong ( $\mathrm{s},>71 \%$ of the tallest signal). High-resolution mass spectrometry (HRMS) was performed using the indicated techniques with a micromass LCT orthogonal time-of-flight mass spectrometer with leucine-enkephalin (TyrGly-Phe-Leu) as an internal lock mass. For UV/Vis measurements, a Shimadzu UV-1800 UV spectrophotometer was used. Melting points were recorded with a Stuart SMP10 melting point apparatus and are uncorrected. Continuous-flow experiments were performed with a Vapourtec E-Series system equipped with a UV150 photoreactor in combination with a high-power LED emitting light at $365 \mathrm{~nm}$ wavelength.

\section{Synthesis of (E)-2-(3-Acetyl-3-methyltriaz-1-en-1-yl)benzoic Acid $(3)^{13}$}

A suspension of 2-aminobenzyl alcohol $(600 \mathrm{mg}, 4.8 \mathrm{mmol}, 1.00$ equiv) in $\mathrm{H}_{2} \mathrm{O}(4 \mathrm{~mL})$ was stirred at $0{ }^{\circ} \mathrm{C}$. Concd $\mathrm{HCl}(2.4 \mathrm{~mL}, 29 \mathrm{mmol}$, 6.00 equiv) was added slowly, resulting in an orange solution. $\mathrm{NaNO}_{2}$ (342 mg, $4.9 \mathrm{mmol}, 1.03$ equiv) in $\mathrm{H}_{2} \mathrm{O}(1 \mathrm{~mL}$ ) was subsequently added dropwise and the orange solution was stirred for a further $20 \mathrm{~min}$ utes before being added dropwise to a stirred solution of $\mathrm{MeNH}_{2}(40 \%$ in $\mathrm{H}_{2} \mathrm{O}, 7.2 \mathrm{~mL}, 92.2 \mathrm{mmol}, 19.2$ equiv) at $-25^{\circ} \mathrm{C}$. The reaction mixture was extracted immediately upon complete addition of the diazonium solution with EtOAc $(2 \times 40 \mathrm{~mL})$. The organic phases were then washed with sodium bicarbonate $(40 \mathrm{~mL})$, brine $(40 \mathrm{~mL})$ and dried over $\mathrm{Na}_{2} \mathrm{SO}_{4}$. The solution was then concentrated in vacuo, providing a red/orange oil, which was used in the next step without further purification.

$\mathrm{CH}_{2} \mathrm{Cl}_{2}$ (50 mL) was added to the red/orange oil and the solution stirred at $0{ }^{\circ} \mathrm{C}$ before Huenig's base was added ( $3.3 \mathrm{~mL}, 19.2 \mathrm{mmol}, 4.0$ equiv). $\mathrm{AcCl}(0.34 \mathrm{~mL}, 4.8 \mathrm{mmol}, 1.0$ equiv) was then added slowly and the solution was stirred for $2.5 \mathrm{~h}$. The reaction mixture was then diluted with $\mathrm{CH}_{2} \mathrm{Cl}_{2}$ and acidified to $\mathrm{pH} 4-5$ with $1 \mathrm{M} \mathrm{HCl}$. The organic phase was separated, washed with brine $(40 \mathrm{~mL})$, dried over $\mathrm{Na}_{2} \mathrm{SO}_{4}$, and then concentrated to provide an orange oil that solidified to give an orange solid that was used without further purification. The orange solid was dissolved in acetone $(50 \mathrm{~mL})$ and stirred at room temperature. $\mathrm{KMnO}_{4}(1.07 \mathrm{~g}, 6.78 \mathrm{mmol}, 1.4$ equiv) was added in portions and the reaction was stirred for a further $3.5 \mathrm{~h} . \mathrm{Na}_{2} \mathrm{SO}_{3}$ (sat. aq.) was added in 2-3 $\mathrm{mL}$ portions until the purple color disappeared. The reaction solution was diluted with $\mathrm{CH}_{2} \mathrm{Cl}_{2}(50 \mathrm{~mL})$, and the solution was acidified to $\mathrm{pH} 4-5$ with $1 \mathrm{M} \mathrm{HCl}$. The organic phase was then separated before being washed with brine $(40 \mathrm{~mL})$ and dried over $\mathrm{Na}_{2} \mathrm{SO}_{4}$. The reaction mixture was then concentrated in vacuo to provide $\mathbf{3}$ as a light-brown solid. The solid was cooled before being triturated from $\mathrm{Et}_{2} \mathrm{O}$ and filtered under suction to yield (E)-2-(3-acetyl-3-methyltriaz-1-en-1-yl)benzoic acid (616 mg, $2.8 \mathrm{mmol}, 58$ \%) (3) as a darkyellow solid ( $\left.\mathrm{mp} 160-162{ }^{\circ} \mathrm{C}\right)$.

IR (neat): 2917 (w), 2734 (w), 1715 (s), 1594 (m), 1484 (m), 1464 (m), $1420(\mathrm{~m}) \mathrm{cm}^{-1}$.

${ }^{1} \mathrm{H} \mathrm{NMR}\left(\mathrm{CDCl}_{3}, 400 \mathrm{MHz}\right): \delta=8.34(\mathrm{~d}, J=8.0 \mathrm{~Hz}, 1 \mathrm{H}), 7.76(\mathrm{~d}, J=8.0$ $\mathrm{Hz}, 1 \mathrm{H}), 7.66-7.62(\mathrm{~m}, J=8.0 \mathrm{~Hz}, 1 \mathrm{H}), 7.55-7.51(\mathrm{~m}, J=8.0 \mathrm{~Hz}, 1 \mathrm{H})$, $3.51(\mathrm{~s}, 3 \mathrm{H}), 2.64(\mathrm{~s}, 3 \mathrm{H})$. 
${ }^{13} \mathrm{C}$ NMR $\left(\mathrm{CDCl}_{3}, 101 \mathrm{MHz}\right): \delta=172.4(\mathrm{C}), 165.9(\mathrm{C}), 146.3(\mathrm{C}), 133.9$ (C), $133.1(\mathrm{CH}), 130.0(\mathrm{CH}), 125.1(\mathrm{CH}), 116.6(\mathrm{CH}), 28.5\left(\mathrm{CH}_{3}\right), 22.0$ $\left(\mathrm{CH}_{3}\right)$.

HRMS (TOF-ESI+): $m / z[\mathrm{M}+\mathrm{H}]^{+}$calcd for $\mathrm{C}_{10} \mathrm{H}_{12} \mathrm{~N}_{3} \mathrm{O}_{3}: 222.0873$; found: 222.0869 .

$\operatorname{UV} / \operatorname{Vis}\left(\lambda_{\max }, \mathrm{MeCN}\right): 288 \mathrm{~nm}$.

Crystal data: (CCDC-2051221): $\mathrm{C}_{10} \mathrm{H}_{11} \mathrm{~N}_{3} \mathrm{O}_{3}$; monoclinic; $a=$ 5.3717(4), $b=15.2445(12), c=12.5898(8) \AA, \beta=91.091(3)^{\circ} ; V=$ $1030.8(2) \AA^{3} ; Z=4$; space group $\mathrm{P} 2 / 1 / \mathrm{n} ; T=120 \mathrm{~K} ; R_{1}=0.052$.

\section{Synthesis of 6a-b; General Procedure}

(E)-2-(3-Acetyl-3-methyltriaz-1-en-1-yl)benzoic acid (0.22 mmol, $0.049 \mathrm{~g}, 1.0$ equiv), and the corresponding azide substrate $(0.44$ $\mathrm{mmol}, 2.0$ equiv) were dissolved in $\mathrm{MeCN}(9 \mathrm{~mL}, 50 \mathrm{mM})$. The homogeneous stock solution was then passed through the $10 \mathrm{~mL}$ coil (3.33 $\mathrm{mL} / \mathrm{min}$, residence time $3.0 \mathrm{~min}$ ) of a Vapourtec E-series photoflow reactor that housed an LED emitting at $365 \mathrm{~nm}$. The temperature was kept constant between $28-30{ }^{\circ} \mathrm{C}$, and pressure was maintained at ca. 2 bar using a backpressure regulator. The emerging product solution was collected in a round-bottom flask prior to being subjected to evaporation of the solvent. All reactions were subsequently purified by flash chromatography (EtOAc/ hexanes) to provide the desired product.

\section{1-Benzyl-1-benzo[1,2,3]triazole (6a)}

Isolated yield: $66 \mathrm{mg}$ (0.32 mmol, 73\%); pale-brown solid; mp 113$115^{\circ} \mathrm{C} ; R_{f}=0.3$ (25\% EtOAc/hexanes).

IR (neat): 3063 (w), 3030 (w), 2990 (w), 2935 (w), 1733 (m), 1712 (m), $1496(\mathrm{~m}), 1494(\mathrm{~m}) \mathrm{cm}^{-1}$.

${ }^{1} \mathrm{H} \mathrm{NMR}\left(\mathrm{CDCl}_{3}, 400 \mathrm{MHz}\right): \delta=8.06(\mathrm{~d}, J=8.0 \mathrm{~Hz}, 1 \mathrm{H}), 7.42-7.26(\mathrm{~m}, 8$ $\mathrm{H}), 5.84(\mathrm{~s}, 2 \mathrm{H})$.

${ }^{13} \mathrm{C}$ NMR $\left(\mathrm{CDCl}_{3}, 101 \mathrm{MHz}\right): \delta=146.3(\mathrm{C}), 134.7(\mathrm{C}), 132.8(\mathrm{C}), 128.9$ (2x CH), $128.4(\mathrm{CH}), 127.6(\mathrm{CH}), 127.4(2 \times \mathrm{CH}), 123.9(\mathrm{CH}), 120.0$ (CH), $109.7(\mathrm{CH}), 52.2\left(\mathrm{CH}_{2}\right)$.

HRMS (TOF-ESI+): $m / z[\mathrm{M}+\mathrm{H}]^{+}$calcd for $\mathrm{C}_{13} \mathrm{H}_{12} \mathrm{~N}_{3}: 210.1031$; found: 210.1026 .

\section{2-(Benzo[1,2,3]triazol-1-yl)-1-phenylethan-1-one (6b)}

Isolated yield: $21 \mathrm{mg}$ ( $0.09 \mathrm{mmol}, 56 \%)$; dark-yellow oil; $R_{f}=0.4(40 \%$ EtOAc/hexanes).

IR (neat): 3055 (w), 3029 (w), 2926 (w), 1644 (s), 1466 (m), 1410 (m). ${ }^{1} \mathrm{H} \mathrm{NMR}\left(\mathrm{CDCl}_{3}, 400 \mathrm{MHz}\right): \delta=8.12-8.05(\mathrm{~m}, 3 \mathrm{H}), 7.69-7.65(\mathrm{~m}, 1 \mathrm{H})$, 7.57-7.47 (m, $3 \mathrm{H}), 7.42-7.37$ (m, $2 \mathrm{H}), 6.10$ (s, $2 \mathrm{H})$.

${ }^{13} \mathrm{C} \mathrm{NMR}\left(\mathrm{CDCl}_{3}, 101 \mathrm{MHz}\right): \delta=190.3(\mathrm{C}), 146.1$ (C), 134.5 (C), 134.0 (C), $133.8(\mathrm{CH}), 133.5(\mathrm{CH}), 130.1(\mathrm{CH}), 129.2(\mathrm{CH}), 128.3(\mathrm{CH}), 127.8$ $(\mathrm{CH}), 124.1(\mathrm{CH}), 120.2(\mathrm{CH}), 109.5(\mathrm{CH}), 53.9\left(\mathrm{CH}_{2}\right)$.

HRMS (TOF-ESI+): $m / z[\mathrm{M}+\mathrm{H}]^{+}$calcd for $\mathrm{C}_{14} \mathrm{H}_{11} \mathrm{NaN}_{3} \mathrm{O}: 260.0794$; found: 260.0797 .

\section{Synthesis of 6c-f, 10a-f; General Procedure}

(E)-2-(3-Acetyl-3-methyltriaz-1-en-1-yl)benzoic acid (2.0 equiv) and the corresponding diene/sydnone substrate (1.0 equiv) were dissolved in $\mathrm{MeCN}(0.1 \mathrm{M}, 0.05 \mathrm{M}$ respectively). The homogeneous stock solution was then passed through the $10 \mathrm{~mL}$ coil $(3.33 \mathrm{~mL} / \mathrm{min}$, residence time: $3 \mathrm{~min}$ ) of a Vapourtec E-series photoflow reactor that housed an LED emitting at $365 \mathrm{~nm}$. The temperature was kept constant between $28-30{ }^{\circ} \mathrm{C}$, and pressure was maintained using a back- pressure regulator. The resulting product solution was collected in a round-bottom flask prior to evaporation of the solvent. All reactions were subsequently purified by silica gel chromatography to provide the desired product.

\section{1,4-Dihydro-1,4-methanonaphthalene (6c)}

Isolated yield: $90 \mathrm{mg}(0.63 \mathrm{mmol}, 42 \%)$; colorless oil.

${ }^{1} \mathrm{H} \mathrm{NMR}\left(\mathrm{CDCl}_{3}, 400 \mathrm{MHz}\right): \delta=7.29-7.24(\mathrm{~m}, 2 \mathrm{H}), 7.07-6.96(\mathrm{~m}, 4 \mathrm{H})$, $5.80(\mathrm{~s}, 1 \mathrm{H}), 2.40(\mathrm{~s}, 3 \mathrm{H})$.

${ }^{13} \mathrm{C} \mathrm{NMR}\left(\mathrm{CDCl}_{3}, 101 \mathrm{MHz}\right): \delta=151.8(2 \mathrm{C}), 142.9(2 \mathrm{CH}), 124.2(2 \mathrm{CH})$, $121.5(2 \mathrm{CH}), 70.1\left(\mathrm{CH}_{2}\right), 50.3(2 \mathrm{CH})$.

\section{1-Methyl-1,4-dihydro-1,4-epoxynaphthalene (6d)}

Isolated yield: $31 \mathrm{mg}(0.19 \mathrm{mmol}, 84 \%)$; yellow oil; $R_{f}=0.4(15 \%$ (EtOAc/hexanes).

IR (neat): 3100 (w), 3058 (w), 3032 (w), 2934 (w), 1705 (s), 1744 (s), $1498(\mathrm{~m}) \mathrm{cm}^{-1}$.

${ }^{1} \mathrm{H} \mathrm{NMR}\left(\mathrm{CDCl}_{3}, 400 \mathrm{MHz}\right): \delta=7.21-7.14(\mathrm{~m}, 2 \mathrm{H}), 7.03-6.92(\mathrm{~m}, 3 \mathrm{H})$, $6.76(\mathrm{~d}, J=8.0 \mathrm{~Hz}, 1 \mathrm{H}), 5.62(\mathrm{~d}, J=4.0 \mathrm{~Hz}, 1 \mathrm{H}), 1.92(\mathrm{~s}, 3 \mathrm{H})$.

${ }^{13} \mathrm{C} \mathrm{NMR}\left(\mathrm{CDCl}_{3}, 101 \mathrm{MHz}\right): \delta=151.3(\mathrm{CH}), 150.5(\mathrm{CH}), 145.54(\mathrm{CH})$, $144.3(\mathrm{CH}), 124.9(\mathrm{C}), 124.7(\mathrm{C}), 119.9(\mathrm{C}), 118.7(\mathrm{CH}), 89.3(\mathrm{CH}), 81.8$ (CH), $15.2\left(\mathrm{CH}_{3}\right)$.

HRMS (TOF-ESI+): $m / z[\mathrm{M}+\mathrm{H}]^{+}$calcd for $\mathrm{C}_{11} \mathrm{H}_{11} \mathrm{O}: 159.0804$; found: 159.0805 .

\section{1-(1,4-Epoxynaphthalen-1-yl)ethan-1-one (6e)}

Isolated yield: $24 \mathrm{mg}$ (0.13 mmol, 57\%); pale-yellow oil; $R_{f}=0.3$ (15\% EtOAc/hexanes).

IR (neat): 3083 (w), 3061 (w), 3025 (w), 2977 (w), 1743 (s), 1710 (s) $\mathrm{cm}^{-1}$.

${ }^{1} \mathrm{H} \mathrm{NMR}\left(\mathrm{CDCl}_{3}, 400 \mathrm{MHz}\right): \delta=7.29-7.24(\mathrm{~m}, 2 \mathrm{H}), 7.07-6.96(\mathrm{~m}, 4 \mathrm{H})$, $5.80(\mathrm{~s}, 1 \mathrm{H}), 2.40(\mathrm{~s}, 3 \mathrm{H})$.

${ }^{13} \mathrm{C}$ NMR $\left(\mathrm{CDCl}_{3}, 101 \mathrm{MHz}\right): \delta=205.2(\mathrm{C}), 148.0(\mathrm{CH}), 147.5(\mathrm{CH})$, $143.4(\mathrm{CH}), 142.3(\mathrm{CH}), 125.6(\mathrm{C}), 125.2(\mathrm{C}), 120.6(\mathrm{C}), 119.6(\mathrm{CH}), 95.7$ (CH), $82.3(\mathrm{CH}), 26.8\left(\mathrm{CH}_{3}\right)$.

HRMS (TOF-ESI+): $m / z[\mathrm{M}+\mathrm{H}]^{+}$calcd for $\mathrm{C}_{12} \mathrm{H}_{11} \mathrm{O}_{2}$ : 187.0754; found: 187.0753 .

\section{1-(4-Methyl-1,4-epoxynaphthalen-1-yl)ethan-1-one (6f)}

Isolated yield: $32 \mathrm{mg}(0.16 \mathrm{mmol}, 72 \%)$; yellow oil; $R_{f}=0.4(15 \%$ EtOAc/hexanes).

IR (neat): 3085 (w), 3068 (w), 3028 (w), 2981 (w), 1748 (s), 1705 (s) $\mathrm{cm}^{-1}$.

${ }^{1} \mathrm{H} \mathrm{NMR}\left(\mathrm{CDCl}_{3}, 400 \mathrm{MHz}\right): \delta=7.19(\mathrm{t}, J=8.0 \mathrm{~Hz}, 2 \mathrm{H}), 7.06-6.95(\mathrm{~m}, 3$ $\mathrm{H}), 6.77(\mathrm{~d}, J=8.0 \mathrm{~Hz}, 2 \mathrm{H}), 2.39(\mathrm{~s}, 3 \mathrm{H}), 1.97(\mathrm{~s}, 3 \mathrm{H})$.

${ }^{13} \mathrm{C}$ NMR $\left(\mathrm{CDCl}_{3}, 101 \mathrm{MHz}\right): \delta=205.5(\mathrm{C}), 150.5(\mathrm{CH}), 149.0(\mathrm{CH})$, $145.9(\mathrm{CH}), 143.5(\mathrm{CH}), 125.5$ (C), 124.9 (C), 119.1 (C), 119.0 (C), 95.1 (CH), $89.7(\mathrm{CH}), 26.8\left(\mathrm{CH}_{3}\right), 15.1\left(\mathrm{CH}_{3}\right)$.

HRMS (TOF-ESI+): $m / z[\mathrm{M}+\mathrm{H}]^{+}$calcd for $\mathrm{C}_{13} \mathrm{H}_{13} \mathrm{O}_{2}$ : 201.0910; found: 201.0911.

\section{2,3-Dihydro-1-pyrrolo[ 1,2$]$ indazole (10a) ${ }^{18 d}$}

Isolated yield: $648 \mathrm{mg}$ (4.1 mmol, 68\%); off-white solid; mp 98-100 ${ }^{\circ} \mathrm{C} ; R_{f}=0.3\left(50 \% \mathrm{CH}_{2} \mathrm{Cl}_{2} /\right.$ EtOAC).

IR (neat): 3023 (w), 2983 (w), $2890(w), 1746$ (s), $1713(\mathrm{~s}) \mathrm{cm}^{-1}$. 
${ }^{1} \mathrm{H}$ NMR $\left(\mathrm{CDCl}_{3}, 400 \mathrm{MHz}\right): \delta=7.64(\mathrm{~d}, J=8.0 \mathrm{~Hz}, 1 \mathrm{H}), 7.54(\mathrm{~d}, J=8.0$ $\mathrm{Hz}, 1 \mathrm{H}), 7.23(\mathrm{t}, J=8.0 \mathrm{~Hz}, 1 \mathrm{H}), 7.01(\mathrm{t}, J=8.0 \mathrm{~Hz}, 1 \mathrm{H}), 4.39(\mathrm{t}, J=8.0$ $\mathrm{Hz}, 2 \mathrm{H}$ ), 3.15 (t, $J=8.0 \mathrm{~Hz}, 2 \mathrm{H}$ ), 2.70 (app p, $J=8.0 \mathrm{~Hz}, 2 \mathrm{H}$ ).

${ }^{13} \mathrm{C} \mathrm{NMR}\left(\mathrm{CDCl}_{3}, 101 \mathrm{MHz}\right): \delta=153.7(\mathrm{C}), 138.8(\mathrm{C}), 125.6(\mathrm{CH}), 120.4$ $(\mathrm{CH}), 119.8(\mathrm{CH}), 117.7(\mathrm{CH}), 116.2(\mathrm{C}), 48.9\left(\mathrm{CH}_{2}\right), 25.8\left(\mathrm{CH}_{2}\right), 23.0$ $\left(\mathrm{CH}_{2}\right)$.

HRMS (TOF-ESI+): $m / z[\mathrm{M}+\mathrm{H}]^{+}$calcd for $\mathrm{C}_{10} \mathrm{H}_{11} \mathrm{~N}_{2}$ : 159.0917; found: 159.0916.

Crystal data: (CCDC-2051222): $\mathrm{C}_{10} \mathrm{H}_{10} \mathrm{~N}_{2}$; monoclinic; $a=6.6759(3), b$ $=17.5344(8), c=7.3037(3) \AA, \beta=108.476(2)^{\circ} ; V=810.89(6) \AA^{3} ; Z=4$; space group $\mathrm{P} 2 / 1 \mathrm{n} ; T=120 \mathrm{~K} ; R_{1}=0.054$.

\section{2-Phenyl-2H-indazole (10b) ${ }^{18 \mathrm{~d}}$}

Isolated yield: $32 \mathrm{mg}(0.17 \mathrm{mmol}, 77 \%)$; yellow solid; $82-84^{\circ} \mathrm{C} ; R_{f}=$ 0.4 (10\% EtOAc/hexanes).

IR (neat): 3130 (w), 3054 (w), 1628 (w), 1592 (m), 1518 (s), 1494 (s) $\mathrm{cm}^{-1}$.

${ }^{1} \mathrm{H} \mathrm{NMR}\left(\mathrm{CDCl}_{3}, 400 \mathrm{MHz}\right): \delta=8.40(\mathrm{~d}, J=1.2 \mathrm{~Hz}, 1 \mathrm{H}), 7.89(\mathrm{dd}, J=8.6$, $1.2 \mathrm{~Hz}, 2 \mathrm{H}), 7.80-7.76(\mathrm{~m}, 1 \mathrm{H}), 7.70(\mathrm{~d}, J=8.6 \mathrm{~Hz}, 1 \mathrm{H}), 7.51(\mathrm{dd}, J=$ 8.7, 7.2 Hz, $2 \mathrm{H}$ ), 7.41-7.36 (m, $1 \mathrm{H}), 7.31$ (ddd, $J=8.9,6.6,1.1 \mathrm{~Hz}, 1 \mathrm{H}$ ), 7.14-7.07 (m, $1 \mathrm{H})$.

${ }^{13} \mathrm{C} \mathrm{NMR}\left(\mathrm{CDCl}_{3}, 101 \mathrm{MHz}\right): \delta=149.8(\mathrm{C}), 140.5(\mathrm{C}), 129.6(2 \mathrm{CH}), 127.9$ $(\mathrm{CH}), 126.8(\mathrm{CH}), 122.8(\mathrm{C}), 122.5(\mathrm{CH}), 121.0(2 \mathrm{CH}), 120.4(\mathrm{CH}), 120.4$ (CH), $117.9(\mathrm{CH})$.

HRMS (TOF-ESI+): $m / z[\mathrm{M}+\mathrm{H}]^{+}$calcd for $\mathrm{C}_{13} \mathrm{H}_{11} \mathrm{~N}_{2}$ : 195.0917; found: 195.0928.

\section{2-(p-Tolyl)-2H-indazole (10c) ${ }^{18 \mathrm{~d}}$}

Isolated yield: $38 \mathrm{mg}(0.18 \mathrm{mmol}, 82 \%)$; yellow solid; $99-101{ }^{\circ} \mathrm{C} ; R_{f}=$ 0.4 (10\% EtOAc/hexanes).

IR (neat): 3133 (w), 3054 (w), 2915 (w), 2859 (w), 1627 (w), 1523 (s) $\mathrm{cm}^{-1}$.

${ }^{1} \mathrm{H} \mathrm{NMR}\left(\mathrm{CDCl}_{3}, 400 \mathrm{MHz}\right): \delta=8.37(\mathrm{~s}, 1 \mathrm{H}), 7.81-7.77(\mathrm{~m}, 3 \mathrm{H}), 7.71$ $(\mathrm{dt}, J=8.0,4.0 \mathrm{~Hz}, 1 \mathrm{H}), 7.34-7.30(\mathrm{~m}, 3 \mathrm{H}), 7.12(\mathrm{ddd}, J=8.4,6.8,0.8$ $\mathrm{Hz}, 1 \mathrm{H}), 2.43(\mathrm{~s}, 3 \mathrm{H})$.

${ }^{13} \mathrm{C} \mathrm{NMR}\left(\mathrm{CDCl}_{3}, 100 \mathrm{MHz}\right): \delta=149.6(\mathrm{C}), 138.3(\mathrm{C}), 137.9(\mathrm{C}), 130.1$ $(2 \mathrm{CH}), 126.6(\mathrm{CH}), 122.7(\mathrm{C}), 122.3(\mathrm{CH}), 120.9(2 \mathrm{CH}), 120.3(\mathrm{CH})$, $120.3(\mathrm{CH}), 117.8(\mathrm{CH}), 21.0\left(\mathrm{CH}_{3}\right)$.

HRMS (TOF-ESI+): $m / z[\mathrm{M}+\mathrm{H}]^{+}$calcd for $\mathrm{C}_{14} \mathrm{H}_{13} \mathrm{~N}_{2}: 209.1073$; found: 209.1069.

\section{2-(4-Methoxyphenyl)-2H-indazole (10d) ${ }^{18 \mathrm{~d}}$}

Isolated yield: $48 \mathrm{mg}$ ( $0.23 \mathrm{mmol}, 79 \%)$; yellow solid; $\mathrm{mp} 130-132{ }^{\circ} \mathrm{C}$; $R_{f}=0.4$ (15\% EtOAc/hexanes).

IR (neat): 3137 (w), 3053 (w), 3012 (m), 2837 (m), 1519 (s), 1244 (s) $\mathrm{cm}^{-1}$.

${ }^{1} \mathrm{H} \mathrm{NMR}\left(\mathrm{CDCl}_{3}, 400 \mathrm{MHz}\right): \delta=8.31(\mathrm{~s}, 1 \mathrm{H}), 7.82-7.78(\mathrm{~m}, 3 \mathrm{H}), 7.70$ (dt, $J=8.8,1.2 \mathrm{~Hz}, 1 \mathrm{H}$ ), 7.32 (ddd, $J=8.4,6.4,0.8 \mathrm{~Hz}, 1 \mathrm{H}$ ), 7.11 (ddd, $J=8.4,6.4,0.8 \mathrm{~Hz}, 1 \mathrm{H}), 7.02(\mathrm{~d}, J=8.0 \mathrm{~Hz}, 2 \mathrm{H}), 3.87(\mathrm{~s}, 3 \mathrm{H})$.

${ }^{13} \mathrm{C}$ NMR $\left(\mathrm{CDCl}_{3}, 101 \mathrm{MHz}\right): \delta=159.3(\mathrm{C}), 149.6(\mathrm{C}), 134.1(\mathrm{C}), 126.5$ (CH), $122.7(\mathrm{C}), 122.4(2 \mathrm{CH}), 122.2(\mathrm{CH}), 120.3(\mathrm{CH}), 120.2(\mathrm{CH}), 117.8$ $(\mathrm{CH}), 114.6(2 \mathrm{CH}), 55.6\left(\mathrm{CH}_{3}\right)$.

HRMS (TOF-ESI+): $m / z[\mathrm{M}+\mathrm{H}]^{+}$calcd for $\mathrm{C}_{14} \mathrm{H}_{13} \mathrm{~N}_{2} \mathrm{O}: 225.1022$; found: 225.1024 .

\section{2-(4-Isopropylphenyl)-2H-indazole (10e)}

Isolated yield: $40 \mathrm{mg}$ (0.17 mmol, 70\%); yellow solid; mp 90-92 ${ }^{\circ} \mathrm{C}$; $R_{f}=0.5$ (15\% EtOAc/hexanes).

IR (neat): 3054 (w), 2958 (m), 2867 (w), 1628 (w), 1522 (s) cm-1.

${ }^{1} \mathrm{H} \mathrm{NMR}\left(\mathrm{CDCl}_{3}, 400 \mathrm{MHz}\right): \delta=8.38(\mathrm{~s}, 1 \mathrm{H}), 7.83-7.79(\mathrm{~m}, 3 \mathrm{H}), 7.71$ $(\mathrm{dt}, J=8.8,1.2 \mathrm{~Hz}, 1 \mathrm{H}), 7.40-7.36(\mathrm{~m}, 2 \mathrm{H}), 7.32$ (ddd, $J=8.8,6.8,0.8$ $\mathrm{Hz}, 1 \mathrm{H}), 7.11$ (ddd, $J=8.8,6.8,0.8 \mathrm{~Hz}, 1 \mathrm{H}), 2.99$ (m, $1 \mathrm{H}), 1.31$ (d, $J=$ $8.0 \mathrm{~Hz}, 6 \mathrm{H})$.

${ }^{13} \mathrm{C} \mathrm{NMR}\left(\mathrm{CDCl}_{3}, 101 \mathrm{MHz}\right): \delta=149.7(\mathrm{C}), 148.9(\mathrm{C}), 138.5(\mathrm{C}), 127.5(2$ $\times \mathrm{CH}), 126.6(\mathrm{CH}), 122.7(\mathrm{C}), 122.3(\mathrm{CH}), 121.0(2 \times \mathrm{CH}), 120.3(\mathrm{CH})$, $120.3(\mathrm{CH}), 117.9(\mathrm{CH}), 33.8(\mathrm{CH}), 23.9\left(2 \mathrm{CH}_{3}\right)$.

HRMS (TOF-ESI+): $m / z[M+H]^{+}$calcd for $\mathrm{C}_{16} \mathrm{H}_{17} \mathrm{~N}_{2}: 237.1386$; found: 237.1383.

\section{2-(3,4-Dichlorophenyl)-2H-indazole (10f)}

Isolated yield: $8 \mathrm{mg}$ (0.03 mmol, $18 \%)$; white solid; mp $127-129^{\circ} \mathrm{C}$; $R_{f}=0.3$ (10\% EtOAc/hexanes).

IR (neat): 3131 (w), 3100 (w), 3059 (w), 1629 (w), 1519 (s), 1485 (s) $\mathrm{cm}^{-1}$.

${ }^{1} \mathrm{H} \mathrm{NMR}\left(\mathrm{CDCl}_{3}, 600 \mathrm{MHz}\right): \delta=8.38(\mathrm{~s}, 1 \mathrm{H}), 8.10(\mathrm{~d}, J=4.0 \mathrm{~Hz}, 1 \mathrm{H})$, 7.78-7.75 (m, $2 \mathrm{H}), 7.70(\mathrm{~d}, J=4.0 \mathrm{~Hz}, 1 \mathrm{H}), 7.60(\mathrm{~d}, J=4.0 \mathrm{~Hz}, 1 \mathrm{H})$, 7.34 (ddd, $J=9.6,7.2,1.2 \mathrm{~Hz}, 1 \mathrm{H}$ ), 7.13 (ddd, $J=9.6,7.2,1.2 \mathrm{~Hz}, 1 \mathrm{H}$ ).

${ }^{13} \mathrm{C}$ NMR $\left(\mathrm{CDCl}_{3}, 151 \mathrm{MHz}\right): \delta=150.1(\mathrm{C}), 139.6$ (C), $133.8(\mathrm{C}), 131.8$ (C), $131.2(\mathrm{CH}), 127.5(\mathrm{CH}), 123.1(\mathrm{CH}), 123.0(\mathrm{C}), 122.6(\mathrm{CH}), 120.4$ $(\mathrm{CH}), 120.3(\mathrm{CH}), 119.6(\mathrm{CH}), 118.0(\mathrm{CH})$.

HRMS (TOF-ESI+): $m / z[\mathrm{M}+\mathrm{H}]^{+}$calcd for $\mathrm{C}_{13} \mathrm{H}_{9} \mathrm{Cl}_{2} \mathrm{~N}_{2}: 263.0137$; found: 263.0139 .

\section{Funding Information}

This research has been enabled through funding by SSPC (European Regional Development Fund; 12/RC2275_P2) and instrumentation acquired through a recent Science Foundation Ireland Infrastructure Call 2018 (18/RI/5702). We gratefully acknowledge support from the School of Chemistry at UCD for a PhD scholarship (to CB). Support of our research through a RSC Research Enablement Grant (E20-2998 to $\mathrm{MB})$ is acknowledged.

\section{Supporting Information}

Supporting information for this article is available online at https://doi.org/10.1055/s-0040-1706016.

\section{References}

(1) (a) Gutmann, B.; Cantillo, D.; Kappe, C. O. Angew. Chem. Int. Ed. 2015, 54, 6688. (b) Movsisyan, M.; Delbeke, E. I. P.; Berton, J. K. E. T.; Battilocchio, C.; Ley, S. V.; Stevens, C. V. Chem. Soc. Rev. 2016, 45, 4892. (c) Baumann, M.; Moody, T. S.; Smyth, M.; Wharry, S. Org. Process Res. Dev. 2020, 23, 1802. (d) Dallinger, D.; Gutmann, B.; Kappe, C. O. Acc. Chem. Res. 2020, 53, 1330.

(2) (a) Colella, M.; Nagaki, A.; Luisi, R. Chem. Eur. J. 2020, 26, 19. (b) Baumann, M.; Moody, T. S.; Smyth, M.; Wharry, S. Eur. J. Org. Chem. 2020, 7398.

(3) (a) Sambiago, C.; Noël, T. Trends Chem. 2020, 2, 92. (b) Di Filippo, M.; Bracken, C.; Baumann, M. Molecules 2020, 25, 356. (c) Elliott, L. D.; Knowles, J. P.; Koovits, P. J.; Maskil, K. G.; Ralph, 
M. J.; Lejeune, G.; Edwards, L. J.; Robinson, R. I.; Clemens, I. R.; Cox, B.; Pascoe, D. D.; Koch, G.; Eberle, M.; Berry, M. B.; BookerMilburn, K. I. Chem. Eur. J. 2014, 20, 15226.

(4) Rehm, T. H. ChemPhotoChem 2020, 4, 235.

(5) For selected recent examples, see: (a) Steiner, A.; Roth, P. M. C.; Strauss, F. J.; Gauron, G.; Tekautz, G.; Winter, M.; Williams, J. D.; Kappe, C. O. Org. Process Res. Dev. 2020, 24, 2208. (b) Levesque, F.; Di Maso, M. J.; Narsimhan, K.; Wismer, M. K.; Naber, J. R. Org. Process Res. Dev. 2020, 24, 2935. (c) Williams, J. D.; Nakano, M.; Gerardy, R.; Rincon, J. A.; de Frutos, O.; Mateos, C.; Monbaliu, J.-C. M.; Kappe, C. O. Org. Process Res. Dev. 2019, 23, 78. (d) Alcazar, J.; Abdiaj, I. Bioorg. Med. Chem. 2017, 25, 6190. (e) Cochran, J. E.; Waal, N. Org. Process Res. Dev. 2016, 20, 1533.

(6) (a) Levesque, F.; Seeberger, P. H. Angew. Chem. Int. Ed. 2012, 51, 1706. (b) Kopetzki, D.; Levesque, F.; Seeberger, P. H. Chem. Eur.J. 2013, 19, 5450. (c) Turconi, J.; Griolet, F.; Guevel, R.; Oddon, G.; Villa, R.; Geatti, A.; Hvala, M.; Rossen, K.; Göller, R.; Burgard, A. Org. Process Res. Dev. 2014, 18, 417. (d) Triemer, S.; Gilmore, K.; Vu, G. T.; Seeberger, P. H.; Seidel-Morgenstern, A. Angew. Chem. Int. Ed. 2018, 57, 5525.

(7) For previous reports on generating benzynes in flow, see: (a) Browne, D. L.; Wright, S.; Deadman, B. J.; Dunnage, S.; Baxendale, I. R.; Turner, R. M.; Ley, S. V. Rapid Commun. Mass Spectrom. 2012, 26, 1999. (b) Nagaki, A.; Ichinari, D.; Yoshida, J. J. Am. Chem. Soc. 2014, 136, 12245. (c) Susanne, F.; Martin, B.; Aubry, M.; Sedelmeier, J.; Lima, F.; Sevinc, S.; Piccioni, L.; Haber, J.; Schenkel, B.; Venturoni, F. Org. Process Res. Dev. 2017, 21, 1779. (d) Tan, Z.; Li, Z.; Jin, G.; Yu, C. Org. Process Res. Dev. 2019, 23,31 .

(8) Himenshima, Y.; Sonoda, T.; Kobayashi, H. Chem. Lett. 1983, 12, 1211.

(9) (a) Wenk, H. H.; Winkler, M.; Sander, W. Angew. Chem. Int. Ed. 2003, 42, 502. (b) Takikawa, H.; Nishii, A.; Sakaib, T.; Suzuki, K. Chem. Soc. Rev. 2018, 47, 8030. (c) Pellissier, H.; Santelli, M. Tetrahedron 2003, 59, 701.

(10) Goetz, A. E.; Garg, N. K. J. Org. Chem. 2014, 79, 846; and references therein.
(11) Chang, D.; Zhu, D.; Shi, L. J. Org. Chem. 2015, 80, 5928.

(12) (a) Maki, Y.; Furuta, T.; Suzuki, M. J. Chem. Soc., Perkin Trans. 1979, 1, 553. (b) Maki, Y.; Furuta, T.; Kuzuya, M.; Suzuki, M. J. Chem. Soc., Chem. Commun. 1975, 616.

(13) Gann, A. W.; Amoroso, J. W.; Einck, V. J.; Rice, P. W.; Chambers, J. J.; Schnarr, N. A. Org. Lett. 2014, 16, 2003.

(14) CCDC 2051221 contains the supplementary crystallographic data for this paper. The data can be obtained free of charge from The Cambridge Crystallographic Data Centre via www.ccdc.cam.ac.uk/structures.

(15) For recent examples, see: (a) Bonciolini, S.; Di Filippo, M.; Baumann, M. Org. Biomol. Chem. 2020, 18, 9428. (b) Di Filippo, M.; Baumann, M. Eur. J. Org. Chem. 2020, 6199. (c) Laudadio, G.; Deng, Y.; van der Wal, K.; Ravelli, D.; Nuno, M.; Fagnoni, M.; Guthrie, D.; Sun, Y.; Noël, T. Science 2020, 369, 92.

(16) Briguglio, I.; Piras, S.; Corona, P.; Gavini, E.; Nieddu, M.; Boatto, G.; Carta, A. Eur. J. Org. Chem. 2015, 612.

(17) (a) Zhang, S.-G.; Liang, C.-G.; Zhang, W.-H. Molecules 2018, 23, 2738. (b) Cerecetto, H.; Gerpe, A.; Gonzalez, M.; Aran, V.; de Ocariz, C. Mini-Rev. Med. Chem. 2005, 5, 869. (c) Gaikwad, D. D.; Chapolikar, A. D.; Devkate, C. G.; Warad, K. D.; Tayade, A. P.; Pawar, R. P.; Domb, A. J. Eur. J. Med. Chem. 2015, 90, 707.

(18) (a) Applegate, J.; Turnbull, K. Synthesis 1988, 1011. (b) Azarifar, D.; Ghasemnejad-Borsa, H. Synthesis 2006, 1123. (c) Browne, D. L.; Vivat, J. F.; Plant, A.; Gomez-Bengoa, E.; Harrity, J. P. A. J. Am. Chem. Soc. 2009, 131, 7762. (d) Fang, Y.; Wu, C.; Larock, R. C.; Shi, F. J. Org. Chem. 2011, 76, 8840.

(19) Larock and co-workers observed a similar outcome using 4nitrophenyl sydnone when no $2 \mathrm{H}$-indazole product was isolated, whereas monochlorinated benzene appendages were tolerated, see reference $18 \mathrm{~d}$.

(20) CCDC 2051222 contains the supplementary crystallographic data for this paper. The data can be obtained free of charge from The Cambridge Crystallographic Data Centre via www.ccdc.cam.ac.uk/structures.

(21) The high-power LED system used in this study is available from Vapourtec ( https://www.vapourtec.com/). 